\title{
NORMAL BASES FOR THE SPACE OF CONTINUOUS FUNCTIONS DEFINED ON A SUBSET OF $\mathbb{Z}_{p}$
}

\author{
ANN VERDOODT
}

\begin{abstract}
Let $K$ be a non-archimedean valued field which contains $\mathbb{Q}_{p}$ and suppose that $K$ is complete for the valuation $|\cdot|$, which extends the $p$-adic valuation. $V_{q}$ is the closure of the set $\left\{a q^{n} \mid n=0,1,2, \ldots\right\}$ where $a$ and $q$ are two units of $\mathbb{Z}_{p}, q$ not a root of unity. $C\left(V_{q} \rightarrow\right.$ $K)$ is the Banach space of continuous functions from $V_{q}$ to $K$, equipped with the supremum norm. Our aim is to find normal bases $\left(r_{n}(x)\right)$ for $C\left(V_{q} \rightarrow K\right)$, where $r_{n}(x)$ does not have to be a polynomial.
\end{abstract}

\section{Introduction}

The main aim of this paper is to find normal bases $\left(r_{n}(x)\right)$ for the space of continuous functions on $V_{q}$, where $r_{n}(x)$ does not have to be a polynomial.

Therefore we start by recalling some definitions and some previous results.

Let $E$ be a non-archimedean Banach space over a non-archimedean valued field $L$.

Let $f_{1}, f_{2}, \ldots$ be a finite or infinite sequence of elements of $E$. We say that this sequence is orthogonal if $\left\|\alpha_{1} f_{1}+\cdots+\alpha_{k} f_{k}\right\|=\max \left\{\left\|\alpha_{i} f_{i}\right\|\right.$ : $i=1, \ldots, k\}$ for all $k$ in $\mathbb{N}$ (or for all $k$ that do not exceed the length of the sequence) and for all $\alpha_{1}, \ldots, \alpha_{k}$ in $L$. If the sequence is infinite, it follows that $\left\|\sum_{i=1}^{\infty} \alpha_{i} f_{i}\right\|=\max \left\{\left\|\alpha_{i} f_{i}\right\|: i=1,2, \ldots\right\}$ for all $\alpha_{1}, \alpha_{2}, \ldots$ in $L$ for which $\lim _{i \rightarrow \infty} \alpha_{i} f_{i}=0$. An orthogonal sequence $f_{1}, f_{2}, \ldots$ is called orthonormal if $\left\|f_{i}\right\|=1$ for all $i$.

This leads us to the following definition: 
If $E$ is a non-archimedean Banach space over a non-archimedean valued field $L$, then a family $\left(f_{i}\right)$ of elements of $E$ is a (ortho)normal basis of $E$ if the family $\left(f_{i}\right)$ is orthonormal and also a basis.

An equivalent formulation is (see [1, Propositions 50.4 and 50.6])

If $E$ is a non-archimedean Banach space over a non-archimedean valued field $L$, then a family $\left(f_{i}\right)$ of elements of $E$ is a (ortho)normal basis of $E$ if each element $x$ of $E$ has a unique representation $x=\sum_{i} x_{i} f_{i}$ where $x_{i} \in L$ and $x_{i} \rightarrow 0$ if $i \rightarrow \infty$, and if the norm of $x$ is the supremum of the norms of $x_{i}$.

Let $\mathbb{Z}_{p}$ be the ring of $p$-adic integers, $\mathbb{Q}_{p}$ the field of $p$-adic numbers, and $K$ is a non-archimedean valued field, $K$ containing $\mathbb{Q}_{p}$, and we suppose that $K$ is complete for the valuation $|\cdot|$, which extends the $p$-adic valuation. Let $a$ and $q$ be two units of $\mathbb{Z}_{p}, q$ not a root of unity. We define $V_{q}$ to be the closure of the set $\left\{a q^{n} \mid n=0,1,2, \ldots\right\}$. The set $V_{q}$ has been described in [3]. Let $C\left(V_{q} \rightarrow K\right)$ (resp. $C\left(\mathbb{Z}_{p} \rightarrow K\right)$ ) be the Banach space of continous functions from $V_{q}$ to $K$ (resp. $\mathbb{Z}_{p}$ to $K$ ) equipped with the supremum norm. $\mathbb{N}$ denotes the set of natural numbers, and $\mathbb{N}_{0}$ is the set of natural numbers without zero.

We introduce the following:

If $x$ is an element of $\mathbb{Q}_{p}, x$ can be written in the following way: $x=\sum_{j=-\infty}^{+\infty} a_{j} p^{j}$ where $a_{-i}$ is zero for $i$ sufficiently large $(i \in \mathbb{N}$ ) (see [1, section 3 and section 4]). This is called the Henseldevelopment of the $p$-adic integer $x$. We then define the $p$-adic entire part $[x]_{p}$ of $x$ by $[x]_{p}=\sum_{j=-\infty}^{-1} a_{j} p^{j}$ and we put $x_{n}=p^{n}\left[p^{-n} x\right]_{p}=\sum_{j=-\infty}^{n-1} a_{j} p^{j}(n \in \mathbb{N})$.

We write $m \triangleleft x$, if $m$ is one of the numbers $x_{0}, x_{1}, \ldots$. We then say that " $m$ is an initial part of $x$ " or " $x$ starts with $m$ " (see $[\mathbf{1}$, section 62]).

If $n$ belongs to $\mathbb{N}_{0}, n=\sum_{j=0}^{s} a_{j} p^{j}$ where $a_{s} \neq 0$, then we put $n_{-}=$ $\sum_{j=0}^{s-1} a_{j} p^{j}$. We remark that $n_{-} \triangleleft n$.

In [1, Theorem 62.2], we find the following result which is due to van der Put:

Theorem.

The functions $g_{0}, g_{1}, \ldots$ defined by

$$
\begin{aligned}
g_{n}(x) & =1 & & \text { if } n \triangleleft x, \\
& =0 & & \text { otherwise },
\end{aligned}
$$


form a normal basis for $C\left(\mathbb{Z}_{p} \rightarrow K\right)$. If $f$ is an element of $C\left(\mathbb{Z}_{p} \rightarrow K\right)$, then $f$ can be written as a uniformly convergent series $f(x)=\sum_{k=0}^{\infty} \gamma_{k} g_{k}(x)$ where $\gamma_{0}=f(0)$ and $\gamma_{n}=f(n)-f\left(n_{-}\right)$if $n \in \mathbb{N}_{0}$.

We now survey the content of this paper:

In Theorem 1 of section 2 , our aim is to find a basis $\left(e_{n}(x)\right)$ analogous to van der Put's basis, but with the space $C\left(\mathbb{Z}_{p} \rightarrow K\right)$ replaced by $C\left(V_{q} \rightarrow K\right)$. If $f$ is an element of $C\left(V_{q} \rightarrow K\right)$, then there exist elements $a_{k}$ of $K$ such that $f(x)=\sum_{k=0}^{\infty} a_{k} e_{k}(x)$ where the series on the right-handside is uniformly convergent. We are able to give an expression for the coefficients $a_{k}$.

In Theorem 2 of section 3, we prove the following result:

Define $r_{n}(x)=\sum_{j=0}^{n} c_{n ; j} e_{j}(x), c_{n ; j} \in K, c_{n ; n} \neq 0\left(\left(e_{n}(x)\right)\right.$ as in Theorem 1 below).

Then $\left(r_{n}(x)\right)$ forms a normal basis for $C\left(V_{q} \rightarrow K\right)$ if and only if for all $n\left\|r_{n}\right\|=1$ and $\left|c_{n ; n}\right|=1$.

In Theorem 3 of section 3, we give an extension of Theorem 2:

Let $\left(r_{n}(x)\right)$ be such a sequence which forms a normal basis for $C\left(V_{q} \rightarrow K\right)$, and let $\left(s_{n}(x)\right)$ be a sequence such that $s_{n}(x)=\sum_{j=0}^{n} d_{n ; j} r_{j}(x)$, $d_{n ; j} \in K, d_{n ; n} \neq 0$. Then $\left(s_{n}(x)\right)$ forms a normal basis for $C\left(V_{q} \rightarrow K\right) \Leftrightarrow$ $\left\|s_{n}\right\|=1,\left|d_{n ; n}\right|=1 \Leftrightarrow\left|d_{n ; j}\right| \leq 1,\left|d_{n ; n}\right|=1$.

Acknowledgement. I thank professor Van Hamme for the advice he gave me during the preparation of this paper.

\section{Proof of the first theorem}

We start with some lemmas and some definitions.

\section{Definition.}

If $b$ and $x$ are elements of $\mathbb{Z}_{p}, b \equiv 1(\bmod p)$, then we put $b^{x}=\lim _{n \rightarrow x} b^{n}$.

The mapping: $\mathbb{Z}_{p} \rightarrow \mathbb{Z}_{p}: x \rightarrow b^{x}$ is continuous.

For more details, we refer the reader to [1, section 32$]$.

\section{Notation.}

Take $m \geq 1, m$ the smallest integer such that $q^{m} \equiv 1(\bmod p)$.

We have $1 \leq m \leq p-1$ and $\left(q^{m}\right)^{x}$ is defined for all $x$ in $\mathbb{Z}_{p}$. 


\section{Definition.}

Let $k$ be a natural number prime to $p$. We denote by $\mathbb{Z}_{p}(k)$ the projective limit $\mathbb{Z}_{p}(k)={\underset{\varliminf}{j}}_{\lim }\left(\mathbb{Z} / k p^{j} \mathbb{Z}\right) \cong(\mathbb{Z} / k \mathbb{Z}) \times \mathbb{Z}_{p}$.

In the following lemma we use the fact that $\mathbb{Z}_{p}(m)=(\mathbb{Z} / m \mathbb{Z}) \times \mathbb{Z}_{p}$ to denote an element of $\mathbb{Z}_{p}(m)$ as $x=(r, y)$. Also, if $n \in \mathbb{N}, n=r+m k$ $(0 \leq r<m)$ then the map $n \rightarrow(r, k)$ imbeds $\mathbb{N}$ in $\mathbb{Z}_{p}(m)$.

\section{Lemma 1.}

The mapping $\varphi: \mathbb{Z}_{p}(m) \rightarrow V_{q}:(r, y) \rightarrow a q^{r}\left(q^{m}\right)^{y}$ is a homeomorphism.

The proof of this lemma can be found in $[2$, p. 377].

\section{Corollary.}

If $q \equiv 1(\bmod p)$, i.e. $m=1$, then the mapping: $\mathbb{Z}_{p} \rightarrow V_{q}: x \rightarrow a q^{x}$ is a homeomorphism.

Let $\beta$ be an element of $\mathbb{Z}_{p} \backslash\{0\}$. We want to know the valuation of the $p$-adic integer $\left(q^{m}\right)^{\beta}-1$. Therefore we need two lemmas:

The following lemmas (2 and 3$)$ are proved in [3]:

\section{Lemma 2.}

Let $\alpha$ be an element of $\mathbb{Z}_{p}, \alpha \equiv 1\left(\bmod p^{r}\right), \alpha \not \equiv 1\left(\bmod p^{r+1}\right) r \geq 1$.

If $(p, r) \neq(2,1), \beta \in \mathbb{Z}_{p} \backslash\{0\}$ then $\alpha^{\beta} \equiv 1\left(\bmod p^{r+\operatorname{ord}_{p} \beta}\right), \alpha^{\beta} \not \equiv 1$ $\left(\bmod p^{r+1+\operatorname{ord}_{p} \beta}\right)$.

\section{Corollary.}

Let $q^{m} \equiv 1\left(\bmod p^{k_{0}}\right), q^{m} \not \equiv 1\left(\bmod p^{k_{0}+1}\right)$. If $\left(p, k_{0}\right) \neq(2,1), \beta \in$ $\mathbb{Z}_{p} \backslash\{0\}$ then $\left(q^{m}\right)^{\beta} \equiv 1\left(\bmod p^{k_{0}+\operatorname{ord}_{p} \beta}\right),\left(q^{m}\right)^{\beta} \not \equiv 1\left(\bmod p^{k_{0}+1+\operatorname{ord}_{p} \beta}\right)$.

In Lemma 2 we excluded the case where $(p, r)=(2,1)$. This case will be handled in the following lemma:

\section{Lemma 3.}

Let $\alpha$ be an element of $\mathbb{Z}_{2}, \alpha \equiv 3(\bmod 4)$. Define a natural number $n$ by $\alpha=1+2+2^{2} \varepsilon, \varepsilon=\varepsilon_{0}+\varepsilon_{1} 2+\varepsilon_{2} 2^{2}+\ldots, \varepsilon_{0}=\varepsilon_{1}=\cdots=\varepsilon_{n-1}=1$, $\varepsilon_{n}=0$.

If $\beta \in \mathbb{Z}_{2} \backslash\{0\}, \operatorname{ord}_{2} \beta=0$ then $\alpha^{\beta} \equiv 1(\bmod 2), \alpha^{\beta} \not \equiv 1(\bmod 4)$. 
If $\beta \in \mathbb{Z}_{2} \backslash\{0\}, \operatorname{ord}_{2} \beta=k \geq 1$ then $\alpha^{\beta} \equiv 1\left(\bmod 2^{n+2+\operatorname{ord}_{2} \beta}\right), \alpha^{\beta} \not \equiv 1$ $\left(\bmod 2^{n+3+\operatorname{ord}_{2} \beta}\right)$.

\section{Corollary.}

If $q \equiv 3(\bmod 4)$, we define a natural number $N$ by $q=1+2+2^{2} \varepsilon$, $\varepsilon=\varepsilon_{0}+\varepsilon_{1} 2+\varepsilon_{2} 2^{2}+\ldots, \varepsilon_{0}=\varepsilon_{1}=\cdots=\varepsilon_{N-1}=1, \varepsilon_{N}=0$.

If $\beta \in \mathbb{Z}_{2} \backslash\{0\}, \operatorname{ord}_{2} \beta=0$ then $q^{\beta} \equiv 1(\bmod 2), q^{\beta} \not \equiv 1(\bmod 4)$.

If $\beta \in \mathbb{Z}_{2} \backslash\{0\}, \operatorname{ord}_{2} \beta=k \geq 1$ then $q^{\beta} \equiv 1\left(\bmod 2^{N+2+\operatorname{ord}_{2} \beta}\right), q^{\beta} \not \equiv 1$ $\left(\bmod 2^{N+3+\operatorname{ord}_{2} \beta}\right)$.

We remark that is possible to write each $x$ and element of $V_{q}$ in the following way: $x=a q^{i_{x}}\left(q^{m}\right)^{\alpha_{x}}$ where $i_{x}$ is a natural number, $0 \leq i_{x}<$ $m$, and where $\alpha_{x}$ is an element of $\mathbb{Z}_{p}$. This immediately follows from Lemma 1. This leads us to the following definition:

\section{Definition.}

We now define a sequence of functions $e_{k}$ in the following way. Write $k(\in \mathbb{N})$ in the form $k=i+m j, 0 \leq i<m(i, j \in \mathbb{N})$. The functions $e_{k}$ are defined by

$$
\begin{aligned}
e_{k}(x)=e_{i+m j}(x) & =1 & & \text { if } x=a q^{i_{x}}\left(q^{m}\right)^{\alpha_{x}} \text { where } i_{x}=i, j \triangleleft \alpha_{x} \\
& =0 & & \text { otherwise. }
\end{aligned}
$$

Let us use the notation $B\left(b, r^{-}\right)$for the 'open' disc with radius $r$ and with center $b$, i.e. $B\left(b, r^{-}\right)=\left\{x \in V_{q}|| x-b \mid<r\right\}$, and $B(b, r)$ for the 'closed' disc with radius $r$ and with center $b$, i.e. $B(b, r)=\left\{x \in V_{q}|| x-b \mid \leq r\right\}$.

In the following lemmas we will show that the functions $e_{k}(x)$ are characteristic functions of discs. There exists a $k_{0}$ such that $q^{m} \equiv 1$ $\left(\bmod p^{k_{0}}\right), q^{m} \not \equiv 1\left(\bmod p^{k_{0}+1}\right)$. We distinguish two cases: $\left(p, k_{0}\right) \neq$ $(2,1)($ Lemma 4$)$, and $\left(p, k_{0}\right)=(2,1)$ i.e. $q \equiv 3(\bmod 4)($ Lemma 5$)$. If we use the same notation in Lemmas 4 and 5 as in the definition, we have

\section{Lemma 4.}

Let $q^{m} \equiv 1\left(\bmod p^{k_{0}}\right), q^{m} \not \equiv 1\left(\bmod p^{k_{0}+1}\right)$ and suppose $\left(p, k_{0}\right) \neq$ $(2,1)$.

If $0 \leq i<m$ then $e_{i}(x)$ is the characteristic function of the closed disc $B\left(a q^{i}, p^{-k_{0}}\right)$, and if $0 \leq i<m, j \geq 1$ then $e_{k}(x)=e_{i+j m}(x)$ is the characteristic function of the open disc $B\left(a q^{i}\left(q^{m}\right)^{j},\left(\frac{p^{-k_{0}}}{j}\right)^{-}\right)$. 


\section{Proof:}

Let $j=\sum_{i=0}^{s} a_{i} p^{i}$ be the Henseldevelopment of $j \in \mathbb{N}_{0}$, with $a_{s}$ different from zero.

If we use the notation $x=a q^{i_{x}}\left(q^{m}\right)^{\alpha_{x}}\left(0 \leq i_{x}<m\right)$ for an element $x$ of $V_{q}$, we will show the following:

a) if $0 \leq i<m:\left|x-a q^{i}\right| \leq p^{-k_{0}}$ if and only if $i_{x}=i$.

b) if $0 \leq i<m, j \geq 1:\left|x-a q^{i}\left(q^{m}\right)^{j}\right|<\frac{p^{-k_{0}}}{j}$ if and only if $i_{x}=i$, $j \triangleleft \alpha_{x}$.

We first prove a). If $i_{x}=i$, then $\left|x-a q^{i}\right|=\left|a q^{i_{x}}\left(q^{m}\right)^{\alpha_{x}}-a q^{i}\right|=$ $\left|\left(q^{m}\right)^{\alpha_{x}}-1\right| \leq p^{-k_{0}}$ by the corollary to Lemma 2 .

If $i_{x} \neq i$, then

$$
\begin{aligned}
\left|x-a q^{i}\right| & =\left|a q^{i_{x}}\left(q^{m}\right)^{\alpha_{x}}-a q^{i}\right| \\
& =\max \left\{\left|a q^{i_{x}}\left(q^{m}\right)^{\alpha_{x}}-a q^{i_{x}}\right|,\left|a q^{i_{x}}-a q^{i}\right|\right\}=1,
\end{aligned}
$$

since $\left|a q^{i_{x}}\left(q^{m}\right)^{\alpha_{x}}-a q^{i_{x}}\right| \leq p^{-k_{0}},\left|a q^{i_{x}}-a q^{i}\right|=1$. This proves a).

Now we prove b).

Suppose $i_{x}=i, j \triangleleft \alpha_{x}$. Then $\left|x-a q^{i}\left(q^{m}\right)^{j}\right|=\left|\left(q^{m}\right)^{\alpha_{x}-j}-1\right| \leq$ $p^{-k_{0}-(s+1)}$ by the corollary following Lemma 2 , since $j$ is an initial part of $\alpha_{x}$. Since $j$ is strictly smaller than $p^{(s+1)}$, we conclude that $\left|x-a q^{i}\left(q^{m}\right)^{j}\right|<\frac{p^{-k_{0}}}{j}$.

For the converse, suppose $\left|x-a q^{i}\left(q^{m}\right)^{j}\right|<\frac{p^{-k_{0}}}{j}$. Then we must have that $i_{x}$ equals $i$, since otherwise $\left|x-a q^{i}\left(q^{m}\right)^{j}\right|=1$ :

$$
\begin{aligned}
\left|x-a q^{i}\left(q^{m}\right)^{j}\right| & \left.=\mid a q^{i_{x}}\left(q^{m}\right)^{\alpha_{x}}-a q^{i}\left(q^{m}\right)^{j}\right) \mid \\
& =\max \left\{\left|a q^{i_{x}}\left(q^{m}\right)^{\alpha_{x}}-a q^{i_{x}}\right|,\left|a q^{i_{x}}-a q^{i}\right|,\left|a q^{i}-a q^{i}\left(q^{m}\right)^{j}\right|\right\} \\
& =1
\end{aligned}
$$

since $\left|a q^{i_{x}}\left(q^{m}\right)^{\alpha_{x}}-a q^{i_{x}}\right| \leq p^{-k_{0}},\left|a q^{i}-a q^{i}\left(q^{m}\right)^{j}\right| \leq p^{-k_{0}}$ (corollary to Lemma 2) and $\left|a q^{i_{x}}-a q^{i}\right|=1$ if $i_{x}$ is different from $i$.

So we have $\left|\left(q^{m}\right)^{\alpha_{x}-j}-1\right|<\frac{p^{-k_{0}}}{j}$ and from this it follows that $\left|\left(q^{m}\right)^{\alpha_{x}-j}-1\right| \leq p^{-k_{0}-(s+1)}$ since $j$ is at least $p^{s}$. This means that $\operatorname{ord}_{p}\left(\alpha_{x}-j\right)$ is at least $s+1$ (again by the corollary to Lemma 2) and so we conclude that $j$ is an initial part of $\alpha_{x}$.

\section{Lemma 5.}

If $q \equiv 3(\bmod 4)$, with $q=1+2+2^{2} \varepsilon$, where $\varepsilon=\varepsilon_{0}+\varepsilon_{1} 2+\varepsilon_{2} 2^{2}+\ldots$, $\varepsilon_{0}=\varepsilon_{1}=\cdots=\varepsilon_{N-1}=1, \varepsilon_{N}=0$, then $e_{0}(x)$ is the characteristic 
function of $V_{q}$, and $e_{j}(x)$ is the characteristic function of the open disc $B\left(a q^{j},\left(\frac{2^{-(N+2)}}{j}\right)^{-}\right)$if $j \geq 1$.

Proof:

In this case $m$ equals one and we use the notation $x=a q^{\alpha_{x}}$ for an element $x$ of $V_{q}$.

It is clear that $e_{0}(x)$ is the characteristic function of $V_{q}$.

If $j$ is at least one, we prove: $\left|x-a q^{j}\right|<\frac{2^{-(N+2)}}{j}$ if and only if $j \triangleleft \alpha_{x}$.

Suppose $j \triangleleft \alpha_{x}$. Then $\left|x-a q^{j}\right|=\left|q^{\alpha_{x}-j}-1\right| \leq 2^{-(N+2)-(s+1)}$ (corollary following Lemma 3 ), and since $j$ is strictly smaller than $2^{s+1}$, we conclude $\left|x-a q^{j}\right|<\frac{2^{-(N+2)}}{j}$.

For the converse, suppose $\left|x-a q^{j}\right|<\frac{2^{-(N+2)}}{j}$. Then $\left|q^{\alpha_{x}-j}-1\right|<$ $\frac{2^{-(N+2)}}{j}$ and so $\left|q^{\alpha_{x}-j}-1\right| \leq 2^{-(N+2)-(s+1)}$ since $j$ is at least $2^{s}$. By the corollary to Lemma 3 , we have that $\operatorname{ord}_{2}\left(\alpha_{x}-j\right)$ is at least $s+1$ and so $j$ is an initial part of $\alpha_{x}$.

\section{Corollary.}

The functions $\left(e_{k}(x)\right)$ are continuous functions on $V_{q}$.

In the following theorem we prove that the sequence $\left(e_{k}(x)\right)$ forms a normal basis for $C\left(V_{q} \rightarrow K\right)$. This implies that if $f$ is an element of $C\left(V_{q} \rightarrow K\right)$, there exists elements $a_{k}$ of $K$ such that $f(x)=\sum_{k=0}^{\infty} a_{k} e_{k}(x)$ where the right-hand-side is uniformly convergent. We are able to give an expression for the coefficients $a_{k}$. The proof of this theorem is analogous to the proof of Theorem 62.2 in [1].

\section{Theorem 1.}

The functions $\left(e_{k}(x)\right)$ form a normal basis for $C\left(V_{q} \rightarrow K\right)$. If $f$ is an element of $C\left(V_{q} \rightarrow K\right)$ then $f$ can be written as a uniformly convergent series $f(x)=\sum_{k=0}^{\infty} a_{k} e_{k}(x)$ where

\section{Proof:}

Let $f$ be an element of $C\left(V_{q} \rightarrow K\right)$, and let $a_{k}$ be defined as $a_{k}=$ $f\left(a q^{k}\right)$ if $0 \leq k<m, a_{k}=a_{i+j m}=f\left(a q^{i}\left(q^{m}\right)^{j}\right)-f\left(a q^{i}\left(q^{m}\right)^{j-}\right)$ if $0 \leq i<m, j>0$. 
We first prove that $a_{k}$ tends to zero if $k$ tends to infinity: for all $\varepsilon>0$, there exists a $J$ such that $k>J$ implies $\left|a_{k}\right| \leq \varepsilon$. To prove this, we distinguish two cases:

i) Let $q^{m} \equiv 1\left(\bmod p^{k_{0}}\right), q^{m} \not \equiv 1\left(\bmod p^{k_{0}+1}\right)$, with $\left(p, k_{0}\right) \neq(2,1)$.

Since the function $f$ is continuous on $V_{q}$, it is uniformly continuous on $V_{q}$, and so there exist an $S$, such that $|x-y| \leq p^{-\left(k_{0}+S\right)}$ implies $|f(x)-f(y)|<\varepsilon$. We then put $J=p^{S} m$.

If $k>J$, and $k$ equals $i+j m$ with $0 \leq i<m$, then we have that $j \geq p^{S}$ and so (corollary to Lemma 2) $\left|a q^{i}\left(q^{m}\right)^{j}-a q^{i}\left(q^{m}\right)^{j_{-}}\right|=\left|\left(q^{m}\right)^{j-j_{-}}-1\right| \leq$ $p^{-\left(k_{0}+S\right)}$ and this implies that $\left|a_{k}\right|=\left|f\left(a q^{i}\left(q^{m}\right)^{j}\right)-f\left(a q^{i}\left(q^{m}\right)^{j_{-}}\right)\right|<\varepsilon$.

ii) Let $q \equiv 3(\bmod 4), q=1+2+2^{2} \varepsilon, \varepsilon=\varepsilon_{0}+\varepsilon_{1} 2+\varepsilon_{2} 2^{2}+\ldots$, $\varepsilon_{0}=\varepsilon_{1}=\cdots=\varepsilon_{N-1}=1, \varepsilon_{N}=0$. We remark that $m$ equals one in this case.

Since the function $f$ is continous on $V_{q}$, it is uniformly continuous on $V_{q}$, and so there exist an $S$, such that $|x-y| \leq 2^{-(N+2+S)}$ implies $|f(x)-f(y)|<\varepsilon$. We then put $J=2^{S}$.

If $k>J$, then (corollary to Lemma 3 ) $\left|q^{k}-q^{k_{-}}\right|=\left|q^{k-k_{-}}-1\right| \leq$ $2^{-(N+2+S)}$ and this implies that $\left|a_{k}\right|=\left|f\left(q^{k}\right)-f\left(q^{k_{-}}\right)\right|<\varepsilon$.

We conclude that $a_{k}$ tends to zero if $k$ tends to infinity.

If $f$ is an element of $C\left(V_{q} \rightarrow K\right)$, we introduce a function $g(x)$ defined by $g(x)=\sum_{k=0}^{\infty} a_{k} e_{k}(x)$ with $a_{k}$ as in (*). Since $\left\|a_{k} e_{k}\right\| \leq\left|a_{k}\right| \rightarrow 0$, the series on the right-hand-side converges uniformly, so the function $g$ is continous as a uniformly limit of continuous functions. We can prove that $g\left(a q^{k}\right)=f\left(a q^{k}\right)$ if $0 \leq k<m$ and that $g\left(a q^{i}\left(q^{m}\right)^{j}\right)-g\left(a q^{i}\left(q^{m}\right)^{j_{-}}\right)=$ $f\left(a q^{i}\left(q^{m}\right)^{j}\right)-f\left(a q^{i}\left(q^{m}\right)^{j-}\right)$ for $0 \leq i<m, j>0$. Then we have $g\left(a q^{k}\right)=$ $f\left(a q^{k}\right)$ for all natural numbers $k$ and by continuity, we conclude that $f(x)=g(x)$.

So we have $f(x)=\sum_{k=0}^{\infty} a_{k} e_{k}(x)$, with $a_{k}$ as in $(*)$.

It is clear that $\|f\| \leq \max _{0 \leq k}\left\{\left|a_{k}\right|\right\}$, but we also have $\left|f\left(a q^{k}\right)\right| \leq\|f\|$ and $\left|\left(a q^{i}\left(q^{m}\right)^{j}\right)-f\left(a q^{i}\left(q^{m}\right)^{j-}\right)\right| \leq\|f\|$, so we conclude $\|f\|=\max _{0 \leq k}\left\{\left|a_{k}\right|\right\}$.

Finally we prove the uniqueness of the coefficients.

If $f(x)=\sum_{k=0}^{\infty} a_{k} e_{k}(x)=\sum_{k=0}^{\infty} b_{k} e_{k}(x)$, then $\sum_{k=0}^{\infty}\left(a_{k}-b_{k}\right) e_{k}(x)=0$. So $\max _{0 \leq k}\left\{\left|a_{k}-b_{k}\right|\right\}=0$, from which it follows that $a_{k}=b_{k}$ for all $k$. This proves the theorem. 


\section{More bases for $C\left(V_{q} \rightarrow K\right)$}

We can make more normal bases, using the basis $\left(e_{k}(x)\right)$ of Theorem 1:

\section{Theorem 2.}

Let $\left(e_{n}(x)\right)$ be as above, and define $r_{n}(x)=\sum_{j=0}^{n} c_{n ; j} e_{j}(x), c_{n ; j} \in K$, $c_{n ; n} \neq 0$. Then $\left(r_{n}(x)\right)$ forms a normal basis for $C\left(V_{q} \rightarrow K\right)$ if and only if $\left\|r_{n}\right\|=1$ and $\left|c_{n ; n}\right|=1$ for all $n$.

The proof of this theorem will not be given here, since it is analogous to the proof of Theorem 2 in [3].

\section{Remark.}

An analogous result can be found on the space $C\left(\mathbb{Z}_{p} \rightarrow K\right)$, if we replace the sequence $\left(e_{n}(x)\right)$ by the van der Put basis $\left(g_{n}(x)\right)$ from the introduction.

We can extend Theorem 2 to the following:

\section{Theorem 3.}

Let $\left(r_{n}(x)\right)$ be a sequence as found in Theorem 2, which forms a normal basis for $C\left(V_{q} \rightarrow K\right)$, and let $\left(s_{n}(x)\right)$ be a sequence such that $s_{n}(x)=$ $\sum_{j=0}^{n} d_{n ; j} r_{j}(x), d_{n ; j} \in K, d_{n ; n} \neq 0$.

Then the following are equivalent:

i) $\left(s_{n}(x)\right)$ forms a normal basis for $C\left(V_{q} \rightarrow K\right)$. .

ii) $\left\|s_{n}\right\|=1,\left|d_{n ; n}\right|=1$.

iii) $\left|d_{n ; j}\right| \leq 1,\left|d_{n ; n}\right|=1$.

Proof:

i) $\Leftrightarrow$ ii) follows from Theorem 2, using the expression $r_{n}(x)=$ $\sum_{j=0}^{n} c_{n ; j} e_{j}(x)$, and ii) $\Leftrightarrow$ iii) follows from the fact that $\left(r_{n}(x)\right)$ forms a normal basis.

\section{Examples.}

1) If a sequence $\left(r_{n}(x)\right)$, as found in Theorem 2 , forms a normal basis of $C\left(V_{q} \rightarrow K\right)$, then so does $\left(s_{n}(x)\right)$, where $s_{n}(x)=r_{0}(x)+$ $r_{1}(x)+\cdots+r_{n}(x)$ : apply iii).

2) If we put for $0 \leq i<m$,

$$
\begin{array}{rlrl}
r_{i}(x)=1 & & \text { if } x=a q^{i_{x}}\left(q^{m}\right)^{\alpha_{x}} \text { where } i_{x}=i \\
& =0 & & \text { otherwise }
\end{array}
$$


anf for $k \geq m$ we put

$$
\begin{aligned}
r_{k}(x)=r_{i+m j}(x)(0 \leq i<m)=1 & \text { if } x=a q^{i_{x}}\left(q^{m}\right)^{\alpha_{x}} \\
& \text { where } i_{x}=i, j \nless \alpha_{x} . \\
=0 & \text { otherwise. }
\end{aligned}
$$

then $\left(r_{n}(x)\right)$ forms a normal basis for $C\left(V_{q} \rightarrow K\right)$. We can apply iii) since $r_{i}(x)=e_{i}(x)$ for $0 \leq i<m, r_{k}(x)=e_{i}(x)-e_{k}(x)$ for $k=i+m j, 0 \leq i<m, j>0$. If $f \in C\left(V_{q} \rightarrow K\right)$, then there exists a uniformly convergent expansion of the form $f(x)=\sum_{k=0}^{\infty} c_{k} r_{k}(x)$, where

$$
\begin{aligned}
& c_{k}=c_{i+j m} \\
& =f\left(a q^{i}\left(q^{m}\right)^{j_{-}}\right)-f\left(a q^{i}\left(q^{m}\right)^{j}\right) \text { if } 0 \leq i<m, j>0 \text {, and } \\
& c_{i}=f\left(a q^{i}\right)-\sum_{j=1}^{\infty} c_{i+j m} \quad \text { if } 0 \leq i<m .
\end{aligned}
$$

\title{
References
}

1. W. H. SchikhoF, "Ultrametric Calculus: An Introduction to p-adic Analysis," Cambridge University Press, 1984.

2. A. VERDOODT, Jackson's Formula with remainder in $p$-adic analysis, Indagationes Mathematicae, N.S. 4(3) (1993), 375-384.

3. A. Verdoodt, Normal bases for Non-Archimedean spaces of continuous functions, Publicacions Matemàtiques UAB 37 (1993), 403-427.

\author{
Vrije Universiteit Brussel \\ Faculty of Applied Sciences \\ Pleinlaan 2 \\ B 1050 Brussels \\ BELGIUM
}

Rebut el 9 de Febrer de 1994 